\title{
Phase Relaxation of Electrons in Disordered Conductors
}

\author{
B.L. Altshuler ${ }^{1,2}$, M.E. Gershenson ${ }^{3}$, and I.L. Aleiner ${ }^{4}$ \\ ${ }^{1}$ NEC Research Institute, 4 Independence Way, Princeton, NJ 08540 \\ ${ }^{2}$ Physics Department, Princeton University, Princeton, NJ 08544 \\ ${ }^{3}$ Serin Physics Laboratory, Rutgers University, Piscataway, NJ 08855 \\ ${ }^{4}$ Physics and Astronomy Department, SUNY at Stony Brook \\ Stony Brook, NY 11794
}

\begin{abstract}
Conduction electrons in disordered metals and heavily doped semiconductors at low temperatures preserve their phase coherence for a long time: phase relaxation time $\tau_{\varphi}$ can be orders of magnitude longer than the momentum relaxation time. The large difference in these time scales gives rise to well known effects of weak localization, such as anomalous magnetoresistance. Among other interesting characteristics, study of these effects provide quantitative information on the dephasing rate $1 / \tau_{\varphi}$. This parameter is of fundamental interest: the relation between $\hbar / \tau_{\varphi}$ and the temperature $T$ (a typical energy scale of an electron) determines how well a single electron state is defined. We will discuss the basic physical meaning of $1 / \tau_{\varphi}$ in different situations and its difference from the energy relaxation rate. At low temperatures, the phase relaxation rate is governed by collisions between electrons. We will review existing theories of dephasing by these collisions or (which is the same) by electric noise inside the sample. We also discuss recent experiments on the magnetoresistance of $1 D$ systems: some of them show saturation of $1 / \tau_{\varphi}$ at low temperatures, the other do not. To resolve this contradiction we discuss dephasing by an external microwave field and by nonequilibrium electric noise.
\end{abstract}

\section{Introduction}

Anomalous magnetoresistance in disordered conductors (doped semiconductors and metals) has been recognized for almost 50 years [四]. For a long time this phenomenon has remained a puzzle. The theoretical understanding of the anomalous magnetoresistance emerged as a spinoff of the theory of Anderson localization. It turned out that the correction to the conductivity, which is due to quantum interference at large length scales, is very sensitive to 
weak magnetic fields. The quantum correction itself may be much smaller than the classical conductivity. Nevertheless, the weak field magnetoresistance is dominated by this correction and its basic features (its amplitude, dependence on both magnitude and direction of the magnetic field, etc.) are very different from that of the classical magnetoresistance. Since the quantum correction can eventually drive the system to the Anderson insulator, the regime when this correction is small, is called the weak localization (WL) regime, and the theory of the anomalous magnetoresistance is now a part of the theory of weak localization.

A qualitative physical interpretation of WL is usually based on estimation of the probability $P(t)$ to find a particle at given time $t$ at the same point where it was at time $t=0$. The quantum correction to this return back probability are due to the interference between two amplitudes to return along the same classical path in the opposite directions. The quantum correction is sensitive to the magnetic field that through the loop formed by the classical self-returning path [2]. High sensitivity is associated with the fact that the typical area $S$ of this loop is large. For large loops, the area $S$ is proportional to the length of the path, i.e., to the time $t$ it takes for an electron to circle the loop. Indeed, the motion is diffusive provided $t$ exceeds the elastic mean free time $\tau$. This means that the typical size of the loop is of the order of $(D t)^{1 / 2}$ and $S(t) \simeq D t$, where $D=v_{F}^{2} \tau / d$ is the diffusion constant ( $d$ is the number of dimensions, $v_{F}$ is the Fermi velocity of electrons).

Let us consider DC resistance of an "infinite" system. In this case, there are two length scales which determine the relevant size of the return path. The first one is associated with the magnetic field, while the second is determined by inelastic collisions of electrons.

The effect of the magnetic field on the conductance is a manifestation of the Aharonov - Bohm effect [3]. Let us consider the amplitude $A(t)=\sum_{j} A_{j}(t)$ of the return probability $P(t)=|A(t)|^{2}$, index $j$ here labels the classical path. In the absence of the magnetic field, the two paths $j$ and $\bar{j}$ which differ only by direction are characterized by the same classical action. Hence, in the semiclassical approximation their contribution to $A(t)$ have identical phases. This leads to constructive interference, to an enhancement of $P(t)$ as compared with its classical value $(D t)^{d / 2}$. As a result, this interference reduces diffusion constant $D$ and increases resistance $R$.

The equality of these two phases is a direct consequence of the $T$ - invariance of the system, and is therefore violated by the magnetic field. The difference between the two phases is determined by the magnetic flux encompassed by the returning trajectories $\Phi_{(j)}(t)=$ $H S_{j}(t)$

$$
\phi_{j}-\phi_{\bar{j}}=2 \pi \frac{\Phi_{(j)}}{\Phi_{0}} ; \quad \Phi_{0}=h c / 2 e,
$$

where $S_{j}$ is the directed area swept by the $j$ th trajectory. Thus, the interference contribution associated with $j$ th path acquires the oscillatory factor $\cos \left(2 \pi \Phi_{(j)} / \Phi_{0}\right)$. Since $S_{j}$ are random, all the contributions from trajectories sweeping typical area larger than $\simeq \Phi_{0} / H$ are diminished. As the result, the first characteristic length scale is the magnetic length $L_{H}=\sqrt{\hbar c / e H}$.

Magnetoresistance will be determined by the classical trajectories sweeping the area of the order of $L_{H}^{2}$, provided electrons preserve the phase coherence during the corresponding time $L_{H}^{2} / D$. Elastic collisions do not effect this phase coherence, while inelastic interactions of the electron with environment (other electrons, phonons, etc.) tend to destroy it. One can 
introduce a length scale $L_{\varphi}$ which corresponds to substantial suppression of the coherence. This scale and corresponding time $\tau_{\varphi}=L_{\varphi}^{2} / D$ depends on temperature $T$, and plays an important role in the description of weak localization phenomena. Since the magnetoresistance depends on the ratio of $L_{\varphi}$ and $L_{H}$, and $L_{H}$ can be tuned by changing the magnetic field, magnetoresistance can be used for the study of inelastic collisions and dephasing of electrons at low temperatures.

A conductor can be considered as infinite for our purposes as long as the distance between the leads is much larger than at least $L_{\min }$ which is the smallest of the two characteristic scales $L_{\varphi}$ and $L_{H}$. It does not mean however, that all of the dimensions of the system should be as large. The sample has a dimension $d=1,2,3$ depending on the relation between its transverse dimensions and $L_{\text {min }}$. We can speak about $1 d$ wires $(2 d$ films) when both thickness of the wire $a$ and its width $W$ (thickness of the film $a$ ) is smaller than $L_{\text {min }}$, despite the fact that both $a$ and $W$ are far in excess of the electronic wavelength $k_{F}^{-1}$. Since in this lecture we discuss recent experiments on magnetoresistance of $1 d$ metallic wires and semiconductor structures, we will consider here only the $1 d$ case, though some of the statements are of a more general validity.

The paper is organized as follows. In Section 2, we discuss qualitatively the main theoretical ideas on the dephasing in disordered systems. Readers interested in rigorous derivations are urged to consult Ref. [6]. Section 3 reviews recent observations of the saturation of $\tau_{\varphi}$ at low temperatures Ref. [17]. Authors of Ref. [17] have suggested an explanation based on the dephasing by the zero point motion of electrons. We think that this explanation is erroneous and elaborate on this issue more in Sec. 4. Section 5 describes new experiments in which no saturation was observed, though the values of $\tau_{\varphi}$ were much longer than the universal "cut-off" suggested. As an explanation of the saturation in Ref. [17], proposed in Sec. 6 the dephasing by the external microwave radiation, and we show in Sec. 7 that the microwave radiation can efficiently dephase electron without significant heating.

\section{Inelastic e-e Collisions and Dephasing Rate}

In early papers (see e.g. Ref. [4] ) on the theory of localization, the dephasing rate $1 / \tau_{\varphi}$ was considered to be of the same order as the inelastic collision rate in perfectly clean conductors. The latter can be expressed as the sum of the electron - phonon $1 / \tau_{e-p h} \simeq T^{3} / \Theta_{D}^{2}$ and electron - electron $1 / \tau_{e-e} \simeq T^{2} / E_{F}$ contributions, where $E_{F}$ and $\Theta_{D}$ are the Fermi and Debye energies correspondingly (here and almost everywhere below we put $\hbar=1$ and $k_{B}=1$ ). Even under this assumption, the $e-e$ contribution dominates at low enough temperatures. It became clear later that static disorder strongly enhances the $e-e$ contribution to the inelastic scattering rate [5, 6], while $1 / \tau_{e-p h}$ is less affected [7]. As the result, both dephasing and energy relaxation rates at low temperatures are governed by collisions between electrons.

To recall the main results on the $e-e$ dephasing rate, let us start with a single electron excitation, assuming that $T=0$, and the rest of the electron gas occupies states below the Fermi level. Dependence of $1 / \tau_{e-e}$ on the energy of the excitation (energy of an electron counted from the Fermi level) $\epsilon$ can be determined in a perturbative calculation [5, 6, 8]. The result (Eq.(4.4) of Ref. [6]) can be rewritten through the dimensionless conductance $g(L)$ (conductance measured in units of $e^{2} / h \simeq 1 / 25.8 k \Omega$ ) of a $\mathrm{d}$ - dimensional cube of the 
size $L$

$$
\frac{1}{\tau_{e-e}(\epsilon)}=C_{d} \frac{\epsilon}{g\left(L_{\epsilon}\right)}, \quad L_{\epsilon} \equiv \sqrt{D / \epsilon}
$$

where $C_{d}$ is the dimension-and-coupling-constant-dependent coefficient. For a weakly interacting $1 d$ electron gas $C_{d}=\sqrt{2}$. Eq.(2) can also be rewritten as

$$
\frac{1}{\tau_{e-e}(\epsilon)}=C_{d} \delta_{1}\left(L_{\epsilon}\right)
$$

where $\delta_{1}(L)=\left(L^{d} \nu\right)^{-1}$ is a one-particle mean level spacing in a $d$-dimensional cube of the size $L \cdot \delta_{1}$ is determined by one-particle density of states $\nu$.

There are several interpretations of this result. One of them [6] is based on the concept of the interaction time which becomes much longer in the disordered case due to diffusive rather than ballistic motion of electrons. It is also possible to appeal to statistical properties of exact one-electron wave functions [9, 10, 11, and we outline this interpretation below.

Inelastic rate $1 / \tau_{e-e}(\epsilon)$ is determined by a pair of collisions between electrons with all four energies - two initial $\left(\epsilon>0\right.$ and $\left.\epsilon^{\prime}<0\right)$ and two final $\left(\epsilon-\omega>0\right.$ and $\left.\epsilon^{\prime}+\omega>0\right)$ belonging to the energy strip with the width $2 \epsilon$ centered at the Fermi level, all the energies here are counted from the Fermi level.

Given the typical absolute value $M\left(L, \omega, \epsilon, \epsilon^{\prime}\right)$ of the matrix element for such a collision in a sample with a size $L$, the inelastic rate can be estimated [12, 10] with the help of the Fermi Golden Rule

$$
\frac{1}{\tau_{e-e}} \propto \sum_{0<\omega<\epsilon} \sum_{-\omega<\epsilon^{\prime}<0} \frac{M\left(L, \omega, \epsilon, \epsilon^{\prime}\right)^{2}}{\delta_{1}(L)},
$$

The matrix elements can be represented as integrals of products of four exact one particle wave functions. In a disordered system, these wave functions oscillate randomly in space, and are only weakly correlated with each other. As a result, the matrix elements are random and for $L$ smaller than $L_{\epsilon}$ (0D case), their typical absolute value $M(L)$ turns out to be of the order of $\delta_{1}(L) / g(L)$, where the small factor $g^{-1}$ reflects the weakness of the correlation between the wave functions. (In the limit $g \rightarrow \infty$ Random Matrix Theory is valid; according to this theory, there is no correlation at all between different eigenvectors and the nondiagonal matrix elements vanish.) Each sum in Eq. (四) leads to the factor $\sim \epsilon / \delta_{1}(L)$. As a result, in $0 D$ case $1 / \tau_{e-e}$ can be estimated as 11

$$
\frac{1}{\tau_{e-e}} \simeq \frac{\epsilon^{2}}{g^{2} \delta_{1}(L)}
$$

It increases with $\epsilon$, and at $L=L_{\epsilon}$, the rate $1 / \tau_{e-e}$ becomes of the order of $\delta_{1}\left(L_{\epsilon}\right)$. This estimate corresponds exactly to Eqs. (2) and (3), and it remains valid even for large samples, $L>L_{\epsilon}$, since $1 / \tau_{e-e}$ cannot depend on $L$ in this limit.

When making estimate (5) we assumed that $1 / \tau_{e-e}$ in Eq. (2) is determined by the energy transfer $\omega$ of the order of $\epsilon$. To take into account quasielastic processes with small energy transfer, let us find the dependence of the matrix element on the transmitted energy $\omega$. From comparison of Eqs. (3) and (4) this dependence in $d$-dimensional sample reads

$$
M^{2} \sim \frac{\delta_{1}(L)^{3} \delta_{1}\left(L_{\omega}\right)}{\omega^{2}}=\frac{\delta_{1}(L)^{4} L^{d}}{\omega^{2} L_{\omega}^{d}} \propto \omega^{-2+d / 2} .
$$


This energy dependence of the matrix element reflects the properties of noninteracting disordered system and is not sensitive to the distribution function.

We see from Eq. (6) that the matrix elements diverge when $\omega \rightarrow 0$ for $d<4$. At $T=0$ this divergence is not dangerous because of two summations in Eq. (5). However, the situation changes when the temperature is finite. In this case $|\epsilon \prime|$ in Eq. (5) is determined by $\max \{T, \omega\}$, and at $\omega<T$ summation over $\epsilon$ can be substituted by the factor $T / \delta_{1}(L)$. According to Eq. (6), it means divergence of the sum over $\omega$ in the lower limit for $d=1,2$. Therefore, $1 / \tau_{e-e}$ is ill-defined at finite temperatures and in low dimensions [13].

This is not a catastrophe, though: $1 / \tau_{e-e}$ itself has no physical meaning. When the energy relaxation rate $1 / \tau_{\epsilon}$, (i.e. the inverse time of thermalization of an excitation with energy $\epsilon$ much larger than temperature $T$ ) is considered, the quasielastic processes are not important. Therefore Eqs. (2) and (3 ) give a good estimate of $1 / \tau_{\epsilon}$. The phase relaxation rate $1 / \tau_{\varphi}$ is more delicate and requires additional consideration [14, 6], since it involves the electron with typical energy $T$, which quasielastic scattering rate is divergent.

An additional phase caused by an inelastic collision is just a product of the energy transfer $\omega$ and the time $t$ that passed after the collision. It means that collisions with arbitrary small $\omega$, which give negligible contribution to $1 / \tau_{\epsilon}$, can cause dephasing, provided the phase is detected over a sufficiently long time. It is clear that the typical observation time $t$ just can not be larger than the dephasing time itself $\tau_{\varphi}$. Therefore in cases when $1 / \tau_{(e-e)}$ diverges $(d=1,2)$, the divergence should be cut off by $\omega \sim 1 / \tau_{\varphi}$. As a result, instead of Eqs. (2) and (3) , we obtain a self-consistent equation for $\tau_{\varphi}$ and $L_{\varphi}$ :

$$
\frac{1}{\tau_{\varphi}(T)}=C_{d} \frac{T}{g\left(L_{\varphi}\right)}=C_{d} \delta_{1}\left(L_{\varphi}\right) T \tau_{\varphi}, \quad L_{\varphi} \equiv \sqrt{D \tau_{\varphi}}
$$

Solving Eq. (7) we find [6, 14]

$$
\frac{1}{\tau_{\varphi}}=\left(T^{2} \Delta_{\xi}\right)^{1 / 3}, \quad L_{\varphi}=\xi\left(\frac{\Delta_{\xi}}{T}\right)^{1 / 3}
$$

in $1 D$ case. Here energy $\Delta_{\xi}=D / \xi^{2}=2 \pi^{2} \delta_{1}(L) / g(L)$, has the meaning of the level spacing on the localization length $\xi$ (we assume $g(L) \gg 1$ ). Corresponding result in two dimensions is

$$
\frac{1}{\tau_{\varphi}}=\frac{T}{g} \ln g
$$

Therefore, $\tau_{\varphi} \rightarrow \infty$, when $T \rightarrow 0$. It should be noted that Eq. (7) for $\tau_{\varphi}$, as well as the WL theory as a whole, is valid when and only when $g\left(L_{\varphi}\right)$ is large. The dephasing length $L_{\varphi}$ increases when $T \rightarrow 0$. As soon as it reaches the localization length $\xi$, Eq. (7) can not be used any more: $g(\xi) \sim 1$ by definition. On the other hand, as long as $g\left(L_{\varphi}\right) \gg 1$, the system behaves as a Fermi liquid, since $T \tau_{\varphi} \gg 1$.

It is also important to emphasize that the condition of the validity of the WL approach, $g\left(L_{\varphi}\right) \gg 1$ does not impose any restriction on the total conductance of the wire, $g(L)=$ $g\left(L_{\varphi}\right)\left(L / L_{\varphi}\right)$. E.g., for the samples described in Sec. 5, $g(L) \simeq 3 \times 10^{-3}$, whereas $g\left(L_{\varphi}\right)>3$, so that WL consideration is still applicable.

We presented here a rather simplified interpretation of old results, which were derived rigorously about 15 years ago and were re-derived later in several ways [15, 16]. 


\section{Experiments on Gold Wires}

These old results have been recalled in connection with recent measurement of the WL magnetoresistance of $1 D A u$ wires [17]. From this measurements authors extracted the temperature dependence of the dephasing rate $1 / \tau_{\varphi}(T)$. They have found that $\tau_{\varphi}(T)$, increases with cooling, when $T$ is large enough, but at $T \sim 1 K$ it saturates at a level of about 1 nanosecond.

In fact, such saturation has been observed by many experimental groups. However, this apparent contradiction with the theory was attributed to one of the two reasons. The saturation was explained either by overheating the electrons (due to applied voltage or to external noise) or by scattering of electrons by localized spins. Authors of Ref. [17 have demonstrated experimentally in a convincing and elegant way that both reasons for the $\tau_{\varphi}(T)$ saturation are not applicable for their samples.

First of all, they observed temperature dependence of the resistivity - probably, due to the effects of the interaction between electrons - at $T$ as low as $40 \mathrm{mK}$. The very fact that such dependence does exist is a strong evidence for the electron gas to have the same temperature as the bath. The effect of paramagnetic impurities was ruled out by adding certain concentration of $F e$ into gold and observing how effect of these additional localized spins disappears with cooling due to the Kondo effect (inelastic cross-section on one-channel Kondo impurities $\propto T^{2}$ at $T$ smaller than the Kondo temperature).

These arguments convinced the authors of Ref. [17] that the finite dephasing at $T=0$ is a fundamental and unavoidable consequence of the interaction between electrons. In light of this experimental data the saturation of $\tau_{\varphi}(T)$ at $T \rightarrow 0$ appears to be a problem, so serious, that it inspired several attempts to reconsider the foundations of the theory of disordered conductors in the weak localization regime.

The main puzzle in experiments Ref. [17] is that $\tau_{\varphi}(T)$ saturates when conductance is still very large: $g\left(L_{\varphi}\right) \sim 10^{3}$. It means that corresponding zero-temperature dephasing length $L_{\varphi}(T=0)$ is much smaller than the localization length $\xi$. Therefore, assuming that this relation between $L_{\varphi}$ and $\xi$ always holds, one should conclude that any interaction between electrons rules out localization of quantum states in a weakly disordered wire with a finite cross-section.

The authors of Ref. [17] came up with an analytical estimate of $L_{\varphi}(T=0)=L_{M J W}$. They argued that their experimental data, as well as all other data available are consistent with the estimate, $L_{\varphi}(T=0) \sim L_{M J W}$. For a wire with a thickness $a$ and a width $W$ their expression for $L_{M J W}$ can be rewritten in the form

$$
L_{M J W}=W a k_{F}=N / k_{F}=\xi /\left(k_{F} l\right),
$$

where $k_{F}$ is Fermi wavenumber, $N=W a / k_{F}^{2}$ is the number of channels in the wire, and $l$ is the mean free path of the electrons. Note that in the WL regime $k_{F} l \gg 1$, and the localization length $\xi$ can be estimated as $\xi \sim l N$. Assuming that Eq. (9) gives correct upper limit for $L_{\varphi}$, one concludes that interactions between electrons prevent localization, provided $N>1$ and $k_{F} l>1$.

In fact, one can discuss a possibility that for some reason interaction between electron, in addition to inelastic dephasing, causes static violation of the $T$ - invariance, e.g. orbital 
ferromagnetism. This violation would saturate the temperature dependence of the magnetoresistance. In this hypothetical case, localization is possible and would be similar to the $1 D$ localization of non-interacting electrons in the presence of magnetic field (unitary ensemble). It would mean, though that the localization length does not depend on the magnetic field at all (in contrast to the experimental evidence [23], see below).

Therefore, assuming that the fact that $L_{\varphi}$ saturation is a fundamental law, one ends up with the conclusion that 1D localization either does not exist in WL regime, or it is magnetic-field-independent.

\section{Nyquist - Johnson Noise and Zero-Point Oscillations}

Dephasing caused by the electron - electron quasielastic collisions Eq. (7), which we interpreted through general properties of matrix elements, can be also understood from a slightly different point of view [14]. Instead of thinking about many colliding electrons, we can consider one electron, which while moving around the loop, is subject (in addition to the quenched disordered potential) to a random time and space dependent electric field. This field is created by the rest of the electron gas and is nothing but the equilibrium electric noise inside the conductor. The advantage of this approach is that correlation functions of this field at large times and distances are determined solely by the conductivity of the system.

It turned out to be possible to take the dephasing effect of this noise into account in a non-perturbative way, and to determine the quantum correction to the conductivity $\sigma(H, T)$ as a function of magnetic field and temperature. In $1 D$ case

$$
\delta g\left(L>L_{\varphi}\right) \propto \frac{L_{\varphi}}{L} \frac{1}{\left[\ln A i\left(\frac{\left(W L_{\varphi} H\right)^{2} r}{\varphi_{0}^{2}}\right)\right]^{\prime}},
$$

where $[\ln A i(x)]^{\prime}$ is the logarithmic derivative of the Airy function, $L_{\varphi}$ is a determined by Eq. (8) and $r$ is geometry dependent coefficient of order unity.

Since Eq. (8) is in qualitatively contradiction to the experimental results, authors of Ref. [17] made an attempt to explain dephasing at $T=0$ by zero-point oscillations of the electric field [18], i.e. they have assumed that dephasing is determined by the processes with the energy transfer $\omega$ much larger than temperature. Their consideration resulted in the length scale $L_{M J W}$.

We do not believe that zero point oscillations can cause any dephasing. Indeed, one can naively consider any environment as a set of harmonic oscillators. This set is characterized by the distribution of frequencies and couplings with a given quantum particle [19]. A collision between the particle and an oscillator is inelastic, provided the particle either transfers energy to the oscillator and excites it, or receives energy from the oscillator. The energy of the zeropoint oscillations can not be transferred, since this energy is simply a difference between the ground state energy of the oscillator and the bottom of the harmonic potential. At $T$ much smaller than the frequency of the oscillator $\omega_{I}$, inelastic collisions are impossible: the oscillator is in the ground state, and the particle does not have enough energy to excite the oscillator. Therefore, with probability exponentially close to unity, the collision is elastic and the oscillator has as little chance to cause dephasing as any static impurity. 
We have to address the question again: why is the experimentally observed dephasing length is always (as it is pointed out in Ref. [17]) smaller than $L_{M J W}$ ? The answer is: it is not always the case! In the next section we briefly discuss experiments where dephasing lengths much larger than $L_{M J W}$ have been observed.

\section{New Data on $L_{M J W}$ Decoherence in 1D $\delta$-doped GaAs Wires.}

Recently, new data on the temperature dependence of $L_{\varphi}$ have been obtained for sub-micronwide "wires" fabricated from the $\delta$-doped GaAs structures [23]. In these samples, a single $\delta$-doped layer with concentration of Si donors $N_{D}=5 \times 10^{12} \mathrm{~cm}^{-2}$ is $0.1 \mu \mathrm{m}$ beneath the surface of an undoped GaAs. The 1D wires were fabricated by electron beam lithography and deep ion etching. A 50-nm-thin silver film deposited on top of the structure was used as a "gate" electrode: the electron concentration $n$ and the resistance of the samples can be "tuned" by varying the gate voltage $V_{g}$ (for more details, see [23]). Below we discuss the data obtained for the sample comprising 360 wires connected in parallel; the length $L$ of each wire is $500 \mu \mathrm{m}$, the effective wire width $W=0.05 \mu \mathrm{m}$. Relatively high concentration of carriers ensures that the number of occupied 1D sub-bands is large $(\sim 10)$ in the wires. The mean free path of electrons $l$ increases with $n$ from $17 \mathrm{~nm}$ to $58 \mathrm{~nm} ;\left(k_{F} l \approx 6-30\right.$, where $k_{F}$ is the Fermi wave number). The sample is one-dimensional with respect to the quantum interference effects at low temperatures: $W<L_{\varphi}(T) \leq \xi$. These 1D conductors demonstrate the Thouless crossover (4) from weak localization (WL) to strong localization (SL) with decreasing the temperature [24, 26]; the crossover temperature $T_{0}$ can be varied over a broad range by the gate voltage. In strong magnetic fields, the crossover "shifts" toward lower temperatures; this shift is accompanied with doubling of the localization length, and halving of the hopping activation energy in the SL regime [24, 26].

Both the temperature and magnetic field dependences of the resistance of these samples are consistent with the theory of WL and interaction effects on the "metallic" side of the crossover $\left(T>T_{0}\right)$ [23]. The phase coherence length $L_{\varphi}$ has been estimated from the WL magnetoresistance; the procedure of extraction of $L_{\varphi}$ for 1D conductors has been described in detail in [23, 25]. The dependences $L_{\varphi}(T)$ are shown in Fig. 1 for different values of the gate voltage $V_{g}$. The experimental values of $L_{\varphi}$ are well described Eq. (8). over the whole temperature range that corresponds to the WL regime. The theoretical dependences Eq. (8) are extended in Fig. 1 down to the crossover temperature. The dependence $L_{\varphi}(T)$ do not saturate down to the crossover temperature and the quasiparticle description holds over the whole WL temperature range.

The observed dependences $L_{\varphi}(T)$ argues against the idea of the decoherence due to zeropoint fluctuations of the electric field [17]. Indeed, Eq. (9) implies that a) narrow channels fabricated from 2DEG cannot demonstrate 1D quantum corrections to the conductivity, since for a strip of 2DEG, $L_{M J W}$ simply equals to the trip width $\mathrm{W}$, and $b$ ) the localization-induced crossover should not be observable in such channels $\left(L_{\varphi}\right.$, being limited by $L_{0}=W$, is always

much smaller than $\xi \gg W$ in this case). The existence of the "cut-off" time $L_{M J W}^{2} / D$ would also preclude observation of the interaction-driven 1D crossover with decreasing the 
temperature. Indeed, as soon as $\tau_{\varphi}$ approaches $\tau_{0}$, the broadening of the electron energy levels, $\frac{\hbar}{\tau_{\varphi}}$, becomes temperature-independent. It is worth noting that at $D \hbar / L_{M J W}^{2}>k_{B} T$ the Fermi-liquid description should also break down.

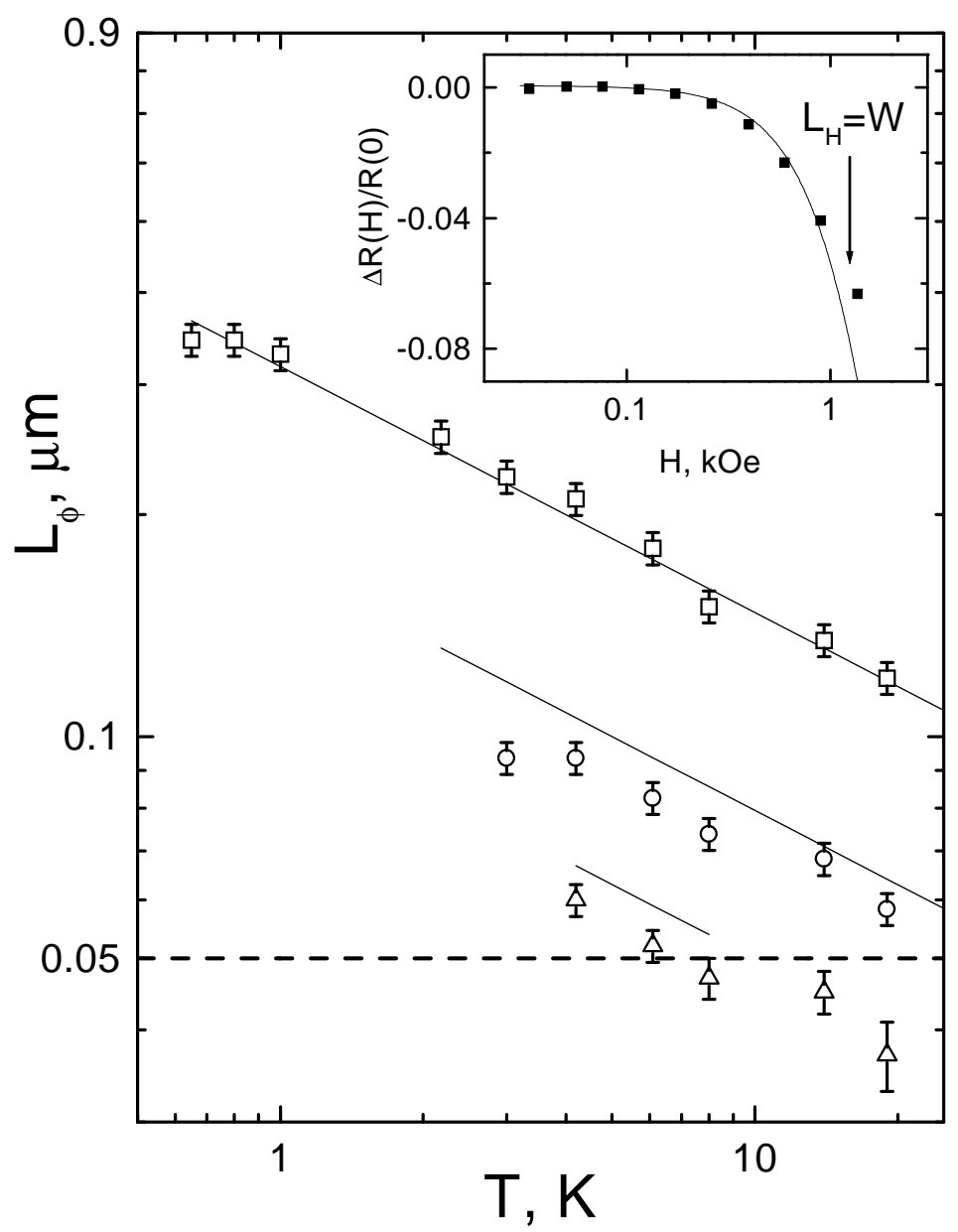

Figure 1: The phase coherence length versus temperature for a $\delta$-doped GaAs wire (for parameters, see text) at different $V_{g}: \square-0.7 \mathrm{~V} ; \bigcirc-0 \mathrm{~V} ; \Delta--0.35 \mathrm{~V}$. Solid lines - Eq. (8), the dashed line - Eq. (9). The insert shows the magnetoresistance at $T=8 \mathrm{~K}, V_{g}=0 \mathrm{~V}$, the solid line - the WL theory fit.

Both consequences of Eq. (9) contradict available experimental data. First, the 1D corrections to the resistance of narrow channels fabricated from 2DEG have been studied for more than a decade. Second, the Thouless crossover has been observed in $\delta$-doped wires with the ratio $\xi / W$ as large as 16 23]. For the data shown in Fig. $1, L_{\varphi}$ near the crossover exceeds the estimate $L_{0}$ by a factor of $\sim 7$ for $V_{g}=0.7 \mathrm{~V}$; hence, the maximum experimental values of $\tau_{\varphi}$ exceed $L_{M J W}^{2} / D$ by a factor of 50 . The experimental values of $\tau_{\varphi}$ an order of 
magnitude greater than the estimate Eq. (9) have been also observed in Ref. [27]. Thus, there is a strong experimental evidence that saturation of the $L_{\varphi}(T)$ dependence is not intrinsic and the mechanism of this saturation is not related to the zero-temperature fluctuations of the electric field.

\section{Dephasing by High Frequency Radiation and Exter- nal Noise}

We believe that saturation of the dependence $L_{\varphi}(T)$ observed in the experiments Ref. [17] is due to phase breaking by the external microwave electromagnetic noise. In order to explain our viewpoint, let us recall another old story about weak localization. Suppose that we have applied to our sample AC electric field (microwave radiation with some frequency $\Omega$ ). The question we want to address is: how will this radiation affect DC conductance and its dependence on magnetic field? Obviously, the radiation can heat the sample, and the temperature dependence of the conductance will transform into its dependence on the amplitude of the $\mathrm{AC}$ electric field $E_{A C}$. However, it turns out that the dephasing effect of radiation can be much more important than this heating [20].

The dephasing effect of the microwave radiation depends on both its amplitude $E_{A C}$ and frequency $\Omega$. According to Ref. [20] this mechanism of dephasing is not effective when frequency is too low or too high. At $\Omega \rightarrow \infty$ one can take the radiation into account using perturbation theory, which gives $1 / \tau_{\varphi} \propto \Omega^{-2}$. The fact that very high-frequency radiation is not effective in dephasing is easy to understand since such field is averaged out in a course of diffusive motion of electron during time $\tau_{\varphi}$.

In the opposite limit of low frequencies one can consider dephasing by the electric field, which is linear in time $E(t) \sim E_{A C} \Omega t$, since the phases, which correspond to two directions of circling the loop remain equal even if a DC field is present. Consider a closed trajectory with the return time $t$. Let us divide this trajectory into small segments $j$; each segment has the length of the order of the elastic mean free path $l$. Let the electron circle around the loop in, say, clockwise direction pass segment $j$ at the time moment $t_{j}$; then, the electron traveling along the same trajectory but in counterclockwise direction will pass the same segment at a moment $t-t_{j}$. Therefore, when passing the same segment in different directions, the electron will acquire the different energies; the difference in this energies $\delta \epsilon_{j}$ can be estimated as

$$
\delta \epsilon_{j}=\alpha_{j} l e\left[E\left(t_{j}\right)-E\left(t-t_{j}\right)\right] \simeq \alpha_{j} e E_{A C} l \Omega\left(2 t_{j}-t\right),
$$

Here $\alpha_{j}$ is a random number which depends on the angle that the electric field makes with the direction of the electron path over the region $j\left\langle\alpha_{j}\right\rangle=0 ;\left\langle\alpha_{i} \alpha_{j}\right\rangle \simeq \delta_{i j}$. In order to find the total energy difference acquired by the electron during the time interval $\left[0 ; t_{j}\right]$, we have to add up contributions from different segments $\epsilon_{j}=\sum_{0<t_{i}<t_{j}} \delta \epsilon_{i}$ and in order to find the total accumulated phase, we have to integrate the energy difference over time

$$
\delta \varphi(t)=\tau \sum_{0<t_{j}<t} \epsilon_{j}=\tau \sum_{0<t_{j}<t} \sum_{0<t_{i}<t_{j}} \delta \epsilon_{i}
$$


Accumulated phase (12) is the random quantity, and, therefore, it is represented by $\left\langle\delta \varphi^{2}\right\rangle$. Substituting Eq. (11) into Eq. (12) and averaging its square with the help of $\left\langle\alpha_{i} \alpha_{j}\right\rangle \simeq \delta_{i j}$, we obtain

$$
\left\langle\delta \varphi(t)^{2}\right\rangle \simeq\left(e E_{A C} l \Omega t\right)^{2} \tau^{2}\left(\frac{t}{\tau}\right)^{3}=D\left(\Omega e E_{A C}\right)^{2} t^{5} .
$$

Determining $\tau_{\varphi}$ from the equation $\left\langle\delta \varphi\left(\tau_{\varphi}\right)^{2}\right\rangle \sim 1$, we find that at small frequencies $1 / \tau_{\varphi}$ increases with $\Omega$ :

$$
\frac{1}{\tau_{\varphi}} \simeq D^{1 / 5}\left(\Omega e E_{A C}\right)^{2 / 5}
$$

All this consideration holds provided $\Omega \tau_{\varphi} \leq 1$. At larger frequencies, as we have already mentioned, the dephasing rate decreases very fast with frequency. Rate $1 / \tau_{\varphi}$ reaches its maximum at a certain frequency $\Omega_{E}$, which is determined by $E_{A C}$

$$
\left[\frac{1}{\tau_{\varphi}(\Omega)}\right]_{\max }=\frac{1}{\tau_{\varphi}\left(\Omega_{E}\right)} \sim \Omega_{E}, \quad \Omega_{E} \approx\left(e^{2} E_{A C}^{2} D\right)^{1 / 3} .
$$

These theoretical predictions have been verified experimentally on inversion channels at the $S i$ surface [21] and on $M g$ films [22]. In this papers it was found that conductance depends substantially on the power of microwave radiation. The frequencies used were $\Omega=9.1 \mathrm{MHz}$ [21] and $\Omega=0.66 \mathrm{GHz}, 3.61 \mathrm{GHz}$ 22]. This dependence had nothing to do with heating (in Ref. [21] the effect had even the opposite sign than that heating would cause) and was in a reasonable agreement with the theory [20].

It is clear that any external noise should have dephasing effect similar to the one of the microwave radiation. We believe that it is external nonequilibrium noise that causes saturation of the dephasing rate in the experiments Ref. [17], while at $T>1 \mathrm{~K}$ the equilibrium (and, thus, temperature-dependent) Nyquist - Johnson noise determines $\tau_{\varphi}$. In the next section we compare dephasing and heating effects of the external noise in the GHz frequency range for a particular case of the $A u$ wires Ref. [17].

\section{Dephasing and heating in $A u$ wires.}

Let us first estimate power of the microwave radiation sufficient for phase breaking at the time scale $\tau_{\varphi}$ in a $1 D$ conductor. We assume that the radiation is in the optimal frequency range $\Omega \tau_{\varphi} \sim 1$. As we have seen, it also means that $\Omega_{E} \tau_{\varphi} \sim 1$ or, according to Eq. (15), $e^{2} E_{A C}^{2} D \tau_{\varphi}^{3} \sim 1$. The radiation dominates dephasing as soon as $1 / \tau_{\varphi}$ given by this estimate exceeds the rate provided by the equilibrium noise $\sim\left(T^{2} D / \xi^{2}\right)^{1 / 3}$, see Eq. (8). For a wire with a length $L$ and a large conductance $g(L) \gg 1$ this happens when $e E>T /[L g(L)]$, since the localization length can be written as $\xi \sim L g(L)$. In terms of the power $P_{A C}=\left(L E_{A C}\right)^{2} / 2 R$, where $R=h\left[e^{2} g(L)\right]^{-1}$ is the resistance of the wire, this inequality takes the form

$$
P_{A C}>P_{\varphi}=R\left(\frac{e k_{B} T}{\hbar}\right)^{2}=\frac{2 \pi}{\hbar g(L)}\left(k_{B} T\right)^{2} .
$$

Here we restore the Planck and Boltzmann constants. Note, that this power is proportional to the total resistance of a wire. Because of a very large resistance of the $\delta$-doped wires studied 
in [24, 26, 23] $\left(R(4 K) \simeq 9 M \Omega\right.$ for a single wire at $V_{g}=0.7 \mathrm{~V}$ in Fig.1), the microwave power required for decoherence in such samples is rather large: $P_{\varphi} \simeq 4 \cdot 10^{-9} \mathrm{~W}$ at $T=1 \mathrm{~K}$. However, for $1 \mathrm{D} \mathrm{Au}$ wires with a small $R \simeq 0.3-1.8 \mathrm{k} \Omega$ [17], $P_{\varphi}$ should be smaller by four orders of magnitude.

Does this microwave power heat the wire? The answer depends on how efficiently the extra energy is removed from the sample. One can propose two mechanisms of cooling: $a$ ) phonon emission and $b$ ) heat flow along the sample into "cold" leads. Let us start with the second mechanism, which is more important at low temperatures even for rather long wires. The expression for the power removed from the wire due to the hot-electron out-diffusion can be obtained using the Wiedemann-Franz law 28. Let $T$ be the temperature of the leads, and $T_{e}$ the temperature of electrons in the wire. (The electron-electron interaction is sufficiently strong in thin metal films at low temperatures to ensure thermalization of electrons and to justify the approximation of local electron temperature [31]). Calculations of the temperature rise for different power levels and sample parameters can be found in Ref. 29]: for a small absorbed power, the difference $\Delta T=T_{e}-T$ has a parabolic profile along the wire, with the peak equal to 1.5 of $\Delta T$ averaged along the wire. The estimate of the heat flow out of both ends of the wire into the "cold" leads for small $\Delta T<<T$ has been done by Prober 28]:

$$
P_{e s} \simeq\left(\frac{2 \pi k_{B} T}{e}\right)^{2} \frac{T \Delta T}{R}=2 \pi g(L) \frac{k_{B}^{2} T \Delta T}{\hbar}
$$

Comparison of Eq. (16) with Eq. (17) shows that at overheating is very small at $P_{A C} \sim P_{\varphi}$ even in the absence of other cooling mechanisms:

$$
\frac{\Delta T}{T}=g(L)^{-2} \ll 1 .
$$

Thus, for any sample with $g(L)>>1$, the microwave radiation can efficiently destroy the phase coherence of the electron wavefunction without heating the electron gas.

In fact, formula (18) even overestimates $\Delta T$ for long wires. In this case, the contribution of the phonon emission becomes dominant. For a sufficiently thin film, the "bottleneck" for the energy transfer from electrons to the thermal bath at low temperatures is formed by the electron-phonon interaction: the non-equilibrium phonons escape ballistically into the substrate [31]. In this case, the rate at which energy flows out of the electron gas by phonon emission, $P_{e-p h}$, is given by the expression:

$$
P_{e-p h}=\frac{C_{e}}{\tau_{e p h}(T)} \Delta T
$$

Here $C_{e}=(a \cdot W \cdot L) \gamma T_{e}$ is the heat capacity of the electron gas in a wire of volume $a \cdot W \cdot L, \gamma$ is the Sommerfield parameter $\left(\simeq 70 \mathrm{~J} / \mathrm{m}^{3} \mathrm{~K}^{2}\right.$ for $\left.\mathrm{Au}\right)$, and $\tau_{\text {eph }}$ is the inelastic electron-phonon scattering time. Equation (19) is valid when $\Delta T=T_{e}-T$, where $T$ is the temperature of equilibrium phonons, is much smaller than both $T_{e}$ and $T$. For an estimate of $\tau_{e p h}$, we can use recent results for the electron-phonon scattering time in thin $A u$ films: $\tau_{e p h}(T) \simeq 1 \mathrm{~ns} \times(1 K / T)^{2}$ [32].

We believe that the experimental results reported in Ref. [17] can be explained by the nonequilibrium external noise. To be specific, let us estimate typical values of $P_{\varphi}, P_{e s}$, 
and $P_{e-p h}$ for sample Au-2 [17]: a gold film (thickness $a=60 \mathrm{~nm}$, width $W=110 \mathrm{~nm}$, length $L=207 \mu \mathrm{m}$ ) with the resistance $R=302 \Omega$ and diffusion constant $D=612 \mathrm{~cm}^{2} / \mathrm{s}$. The temperature dependences of $P_{\varphi}$ as well as $P_{e-p h}$ and $P_{e s}$ calculated for $\Delta T=0.3 T_{p h}$ are shown in Fig. 2; note that $P_{\varphi}$ is in the sub-picowatt range at $T \leq 0.1 K$. At low temperatures, electron diffusion is the process controlling energy flow out of the electron gas, while at higher temperatures the phonon emission dominates the electron gas cooling. For conditions of the experiment [17], balancing of the noise power $P_{\varphi}$, sufficient for phase breaking, by the outcoming power due to phonon emission and hot-electron out diffusion, $P_{e-p h}+P_{e s}$, corresponds to a negligible increase of the electron temperature. In this situation, the rf noise can efficiently destroy the phase coherence of the electron wavefunction without heating the electron gas. This explains why a well-pronounced temperature dependence of the resistance (due to the interaction effects) has been observed at temperatures where the dependence $L_{\varphi}(T)$ was already completely saturated [17].

Table 1: Parameters of the samples studied in Ref. [17], $(A u-1-A u-4)$ and the samples described in Sec. 5, (GaAs). $R$ is the resistance of the sample, $L$ is its length, $g=25.8 \mathrm{k} \Omega / R$ is the dimensionless conductance, $\tau_{\varphi}^{\text {sat }}$ is the experimental saturation value of the dephasing time claimed in Ref. [17], $V_{D C}$ is the measuring DC bias applied to the sample [33], $P_{D C}$ is the corresponding DC power, $V_{\varphi}$ and $P_{\varphi}$ is respectively the $\mathrm{AC}$ voltage and power at optimal frequency $\Omega \simeq 1 / \tau_{\varphi}^{\text {sat }}$ needed to produce $\tau_{\varphi}^{\text {sat }}$, see Eq. (14). $P_{\varphi}$ and $V_{\varphi}$ for $G a A s$ sample not showing saturation is the estimate of the $A C$ power required to affect the observed dependence.

\begin{tabular}{|c|c|c|c|c|c|}
\hline & $A u-1$ & $A u-2$ & $A u-3$ & $A u-4$ & GaAs \\
\hline$R, \Omega$ & 1,687 & 302 & 1,443 & 1,812 & $9 \times 10^{6}$ \\
\hline$L, \mu \mathrm{m}$ & 57.9 & 207 & 155 & 57.9 & 500 \\
\hline$g(L)$ & 15.1 & 84.1 & 17.6 & 14.0 & $2.9 \times 10^{-3}$ \\
\hline$g\left(L_{\varphi}\right)$ & 160 & 1090 & 525 & 225 & $3-10$ \\
\hline$\tau_{\varphi}^{\text {sat }}, n \mathrm{~s}$ & 3.41 & 4.19 & 2.24 & 1.56 & no saturation \\
\hline$V_{D C}, \mu \mathrm{V}$ & 8.37 & 14.57 & 14.68 & 8.64 & 50 \\
\hline$P_{D C}, \mathrm{~W}$ & $4.2 \times 10^{-14}$ & $7.0 \times 10^{-13}$ & $1.5 \times 10^{-13}$ & $4.0 \times 10^{-14}$ & $2.8 \times 10^{-16}$ \\
\hline$V_{\varphi}, \mu \mathrm{V}$ & 2.0 & 2.0 & 8.8 & 6.4 & $2 \times 10^{4}$ \\
\hline$P_{\varphi}, \mathrm{W}$ & $2.4 \times 10^{-15}$ & $1.4 \times 10^{-14}$ & $5.4 \times 10^{-14}$ & $2.2 \times 10^{-14}$ & $>4 \times 10^{-9}$ \\
\hline
\end{tabular}

In fact, the dephasing power $P_{\varphi}$ is even much smaller than the power dissipated by the DC voltage which was applied for the measurements [33] (see Fig. 2). Table 11 presents some parameters of the samples [17] and the noise power in the frequency range $f=4 \times$ $10^{7}-2 \times 10^{8} \mathrm{~Hz}$, which would provide the experimentally observed dephasing rate $1 / \tau_{\varphi}$. One can see that $P_{\varphi}$ for most samples is about an order of magnitude smaller than $P_{D C}$, which is experimentally proven not to heat the wire. 


\section{Conclusion}

In this lecture we argue that there is no need to modify the existing theory of dephasing in disordered conductors due to the electron-electron interactions. We have demonstrated that in $\delta$-doped GaAs structures the theory describes both the temperature and magnetic field dependences of the conductivity in the weak localization regime up to the crossover to the strong localization.

We have proposed a mechanism that may be responsible for saturation of the dephasing rate at $T \rightarrow 0$ in many experiments. This is dephasing by the external non-equilibrium noise. It turns out that the dephasing effect of this noise is much stronger than its heating effect. Therefore, the temperature dependence of the conductivity observed in Ref. [17] does not prove the absence of this noise in the system.

In order to preserve the phase coherence for a long time, one has to reduce the noise amplitude in the frequency range $\Omega \sim 1 / \tau_{\varphi}$ below a very low level. Such reduction could be a difficult technical problem at ultra-low temperatures.

\section{References}

[1] R.A. Chentsov, Zh. Exp. Teor. Fiz., 1 8, 374 (1948).

[2] B.L. Altshuler and P.A. Lee, Physics Today, 41, 36 (1988).

[3] Y. Aharonov and D. Bohm, Phys. Rev. 115, 485 (1959).

[4] D.J.Thouless, Phys. Rev. Lett. 39, 1167 (1977).

[5] A.Schmid, Z. Phys. 271, 251 (1973).

[6] B.L. Altshuler and A.G. Aronov, in Electron-Electron Interaction in Disordered Systems, edited by A.L. Efros and M. Pollak (North-Holland, Amsterdam, 1985).

[7] M. Reyzer and A.V. Sergeev, Sov. Phys. JETP, 65, 616 (1986).

[8] B.L. Altshuler and A.G. Aronov, JETP Lett., 30, 482 (1979).

[9] Ya.M. Blanter, Phys. Rev. B 54, 12807 (1996).

[10] B.L. Altshuler, Y. Gefen, A. Kamenev, L.S. Levitov, Phys. Rev. Lett. 78, 2803 (1997).

[11] V. Prigodin and B.L. Altshuler, preprint cond-mat/9703071.

[12] U. Sivan, Y. Imry and A. Aronov, Europhys. Lett. 28, 115 (1994).

[13] E. Abrahams, P.W. Anderson, P.A. Lee, and T.V. Ramakrishnan, Phys. Rev. B24, 6783 (1981).

[14] B.L. Altshuler, A.G. Aronov, and D.E. Khmelnitskii, J. Phys. C 15, 7367 (1982). 
[15] A. Stern, Y. Aharonov, and Y. Imry, Phys. Rev. A , 41, 3436 (1990).

[16] S. Chakravarty and A. Schmid, Phys. Rep. 140, 193 (1986).

[17] P. Mohanty, E.M.Q. Jarivala, and R.A. Webb, Phys.Rev.Lett, 78, 3366 (1997).

[18] P.Mohanty and R.A. Webb, Phys. Rev. B55, 13452 (1997).

[19] A.O. Caldeira and A.J. Leggett, Phys. Rev. Lett, 46, 211 (1981).

[20] B.L. Altshuler, A.G. Aronov, and D.E. Khmelnitskii, Solid State Commun. 39, 619 (1981).

[21] S.A. Vitkalov, G.M. Gusev, Z.D. Kwon, and G.I. Leviev, JETP Lett. 43, 185 (1986); S.A. Vitkalov, G.M. Gusev, Z.D. Kwon, G.I. Leviev, and V.I. Falko, JETP 67, 1080 (1988).

[22] Shiguang Wang and P.E. Lindelof, Phys. Rev. Lett. 59, 1156 (1987).

[23] Yu. B. Khavin, M. E. Gershenson, and A. L. Bogdanov, to be published.

[24] M. E. Gershenson, Yu. B. Khavin, A. G. Mikhalckuk, H. M. Bozler, and A. L. Bogdanov, Phys.Rev.Lett. 79, 725 (1997).

[25] P. M. Echternach, M. E. Gershenson, H. M. Bozler, A. L. Bogdanov and B. Nilsson, Phys. Rev. B 48, 11516 (1993).

[26] M. E. Gershenson, Yu. B. Khavin, and A. L. Bogdanov, Sov.Phys. - Uspekhi, February 1998.

[27] H. Linke, P. Omling, H. Xu, and P. E. Lindelof, Phys.Rev. B 55, 4061 (1997).

[28] D.E. Prober, Appl. Phys. Lett. 62, 2119 (1993); Phys. Rev. Lett. 75, 3964 (1995).

[29] A. Mittal et al., Surf. Sci. 361/362, 537 (1996).

[30] T. J. Thornton et al., Phys. Rev. Lett. 56, 1198 (1986); S. Wind et al., Phys. Rev. Lett. $\mathbf{5 7 ,} 633(1986)$;

[31] E. M. Gershenzon et al., Sov. Phys.- JETP 59, 442 (1984).

[32] B. Belevtsev et al., Sov. Phys. Low Temp. Phys. 21, 646 (1995); N. G. Ptitsina et al., Phys. Rev. B 56, 10089 (1997).

[33] We are grateful to R.A. Webb for making this data available to us. 


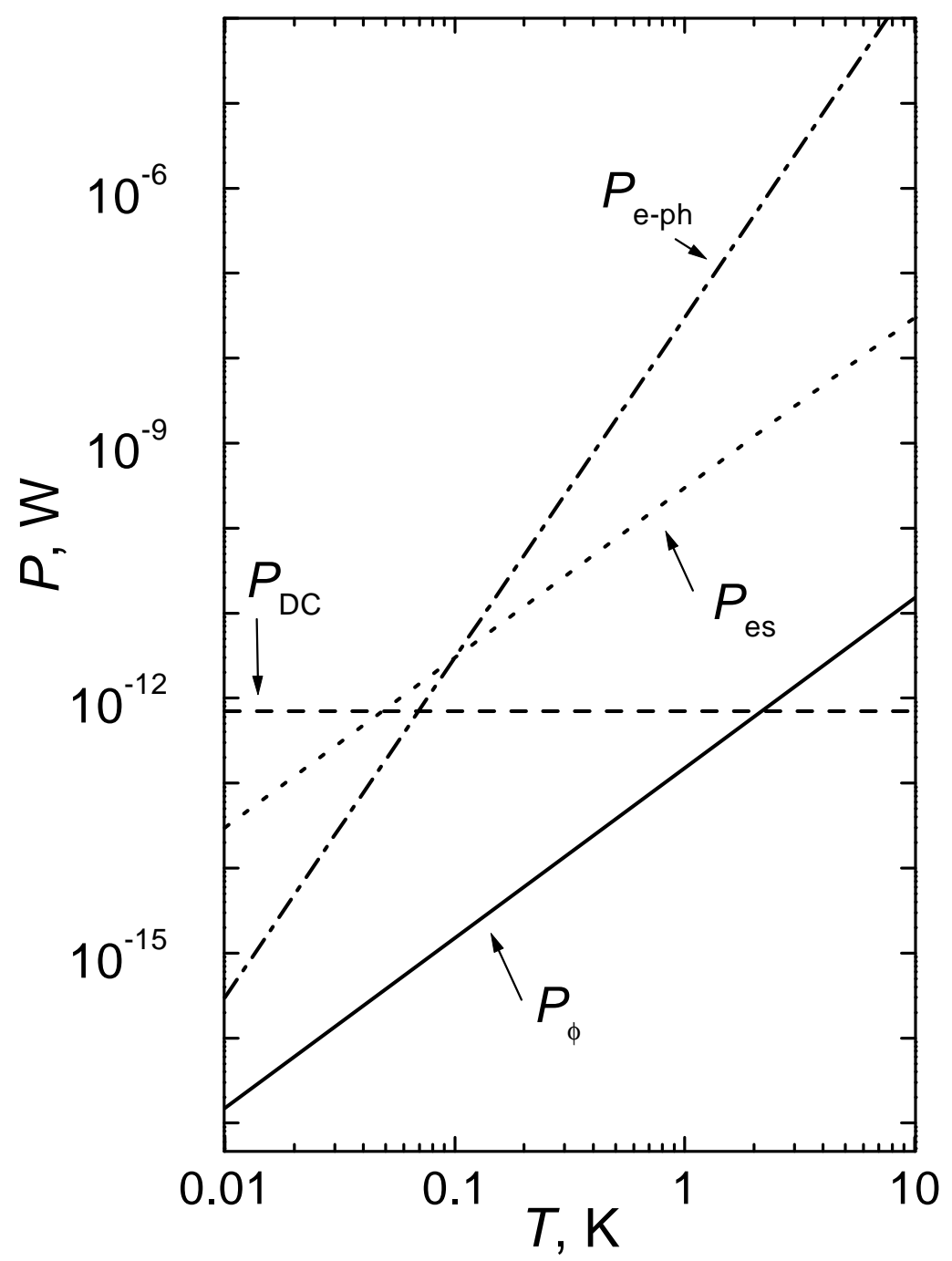

Figure 2: The temperature dependences of $P_{\varphi}, P_{e-p h}$, and $P_{e s}$ for sample Au-2 [16]. The horizontal dashed line is the power of the DC current that has been used in measurements. 